\title{
Etiología y evolución de las infecciones osteo-articulares 2009-2015. Hospital Pediátrico del Centro Hospitalario Pereira Rossell, Uruguay
}

\author{
Carlos Zunino, Alejandra Vomero, Soledad Pandolfo, Claudia Gutiérrez, \\ Gabriela Algorta, M. Catalina Pírez y Gustavo Giachetto
}

\section{Etiology and evolution of the osteoarticular infections. 2009-2015. Pediatric Hospital Pereira Rossell Uruguay}

Background: The most frequent osteoarticular infections (OAI) etiological agent is Staphylococcus aureus. The prevalence of other microorganisms has changed after the introduction of new vaccines. Aim: To describe the etiology and evolution of the OAIs in children hospitalized in Pediatric Hospital Pereira Rossell between 2009 and 2015. Patients and Methods: Joint fluid and bone puncture studies of hospitalized children suspected of OAI. Epidemiological characteristics, isolated microorganisms, complementary tests, initial treatment, complications, and days of hospitalization were analyzed. Results: 335 patients; the etiology was established in 113. Clinical data could be analyzed in 87 cases. The average age was 6.1 years. They corresponded to: methicillin-sensitive $S$. aureus (n: 47), community-acquired methicillin-resistant $S$. aureus (CA-MRSA) (n: 11), Streptococcus pyogenes (n: 6), Streptococcus pneumoniae (n: 1), Haemophilus influenzae type b (n: 2), Gram-negative bacilli (n: 9), Kingella kingae (n: 1) others (n: 6). In 4 cases, more than one microorganism was isolated. CA-MRSA infections were more serious. Discussion: A decrease is observed in the number of CA-MRSA isolates. A fact to be highlighted is the impact of universal vaccination against $S$. pneumoniae and $H$. influenzae type b. It is a finding to be monitored the increasing number of Gram-negative bacilli isolates.

Key words: Staphylococcus aureus; osteoarticular infections; community acquired; methicillin resistant etiology; hospitalized children.

Palabras clave: Staphylococcus aureus; aparato locomotor; adquirido en la comunidad; resistente a meticilina; etiología; niño hospitalizado.

\section{Introducción}

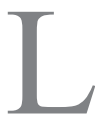
as infecciones osteoarticulares (IOA) agudas constituyen una patología poco frecuente pero potencialmente grave, ya que pueden dejar secuelas y llevar a la muerte ${ }^{1}$. La incidencia varía entre 10 y 25 por 100.000 niños previamente sanos ${ }^{2}$. El diagnóstico requiere un alto índice de sospecha clínica complementándose con estudios imagenológicos.

La confirmación exige el aislamiento del microorganismo causal a través del cultivo del material obtenido de muestras de punción ósea y/o articular. La pesquisa de agentes microbianos en hemocultivo se recomienda sea considerado para el diagnóstico de esta enfermedad en algunas situaciones ${ }^{2,3}$.

En Uruguay, los únicos datos publicados sobre etiología, presentación y evolución clínica de IOA en niños proceden del Hospital Pediátrico del Centro Hospitalario Pereira Rossell (HP-CHPR), centro de referencia de la Administración de Servicios de Salud del Estado (ASSE).
La primera serie comunicada corresponde al período 2003 a 2005 y la segunda 2009 a $2012^{4,5}$. En ambas series, el aislamiento del agente causal en hemocultivo y/o punción ósea o articular no superó el $50 \%$ de los casos; Staphylococcus aureus fue el principal agente etiológico en todos los grupos etarios, coincidiendo con lo descrito en la literatura internacional ${ }^{1,2,6,7}$. En el primer período, se observó el surgimiento de cepas de $S$. aureus resistente a meticilina adquirido en la comunidad (SARM-AC) con mayor morbi-mortalidad, lo que determinó cambios en el abordaje diagnóstico y terapéutico ${ }^{8}$. En el segundo período, se analizó únicamente los casos de IOA causadas por S. aureus. Si bien la prevalencia de este microorganismo fue similar al período anterior, se observó una reducción significativa de la prevalencia de SARM-AC. A pesar de su reducción, la presentación y evolución clínica continúa siendo más grave, con casos mortales y mayor número de secuelas 9 .

La prevalencia de otros agentes bacterianos ha cambiado luego de la introducción de las nuevas vacunas
Universidad de la República. Uruguay.

Facultad de Medicina Clínica Pediátrica $(C Z, A V$, SP, MCP, GG). Hospital Pereira Rossell. Uruguay. Laboratorio de Bacteriología (CG, GA)

Financiamiento: No hubo Conflictos de interés: Inexistentes. Recibido: 8 de febrero de 2017 Aceptado: 26 de abril de 2017

Correspondencia a: Gustavo Giachetto ggiachet@gmail.com 
$\operatorname{conjugadas}^{10}$. Uruguay fue el primer país de Latinoamérica que introdujo en 1994 la vacuna conjugada contra $H$. influenzae tipo b en el Programa Ampliado de inmunizaciones (PAI), demostrando una reducción significativa de las enfermedades invasoras, fundamentalmente meningoencefalitis aguda supurada ${ }^{11}$. En una publicación que analiza la reducción de casos de enfermedad invasora por $H$. influenzae tipo b hospitalizados entre 1993 y 1999 , las IOA representaron el tercer evento más frecuente después de meningitis y neumonía; se registraron infecciones multifocales ${ }^{12}$. En el año 2008 se introdujo la vacunación universal con vacuna neumocóccica conjugada 7 valente (VNC 7), sustituida por VNC 13 valente en el 2010. Se evidenció una efectividad vacunal de $95 \%$ frente a la enfermedad invasora causada por S. pneumoniae en niños con dos dosis ${ }^{13}$. Otros estudios muestran una reducción significativa sobre la carga de neumonía adquirida en la comunidad y las hospitalizaciones por neumonía y meningitis en el HP-CHPR ${ }^{14-16}$. No se dispone de datos nacionales acerca del impacto de la vacunación sobre las IOA.

En los últimos años otros microorganismos han cobrado importancia como causa de IOA, entre ellos Kingella kingae, especialmente en niños bajo 5 años de edad. En Uruguay, en el año 2012 se comunican los dos primeros casos de aislamiento de este microorganismo en niños bajo 2 años de edad en una institución de asistencia privada ${ }^{17}$.

Es fundamental continuar con la vigilancia de la etiología y el patrón de susceptibilidad antimicrobiana y la correlación clínica-evolutiva para profundizar en el conocimiento de esta enfermedad y optimizar su abordaje diagnóstico y terapéutico.

\section{Objetivo}

Describir la etiología y evolución de los casos de IOA en niños de 0 a 14 años de edad, internados en el HP-CHPR entre el 1 de enero de 2009 y 31 de diciembre de 2015. Comparar los hallazgos etiológicos con los del período 1 de diciembre de 2003 a 31 de diciembre de 2005 .

\section{Pacientes y Métodos}

Se realizó un estudio descriptivo, retrospectivo, en el que incluyeron pacientes bajo 15 años de edad, con sospecha de IOA, hospitalizados en el período de 7 años antes indicado, en los que se aisló un agente bacteriano del tejido óseo y/o líquido articular y se tuvo acceso a su historia clínica. Se consideraron síntomas o signos de sospecha de IOA: dolor metafisario u osteoarticular, limitación funcional, edema, signos inflamatorios y fiebre. Se consideró caso sospechoso aquel con dichas manifestaciones clínicas y hallazgos imagenológicas compatibles y/o hemocultivo positivo. Se consideró IOA confirmada aquellos casos con aislamiento del microorganismo causal en muestras de punción ósea y/o articular ${ }^{3}$.

La identificación de los pacientes se realizó a partir de la base de datos informatizada del Laboratorio de Microbiología del HP-CHPR mediante el análisis de todas las muestras de punciones óseas y articulares procesadas en dicho período.

El cultivo de las muestras y la identificación de los agentes fue realizado mediante técnicas microbiológicas habituales ${ }^{18}$. El estudio de la susceptibilidad microbiana in vitro se realizó por el método de disco difusión de Kirby Bauer. A partir del año 2010 se utilizó para la identificación y estudio de susceptibilidad el método automatizado Vitek2 System ${ }^{\circledR}$, Biomerieux.

Se describió edad, género, topografía de la infección, microorganismo, susceptibilidad antimicrobiana, reactantes de fase aguda al ingreso (recuento leucocitario, concentración plasmática de proteína $\mathrm{C}$ reactiva-PCR) y hallazgos en estudios imagenológicos. Se evaluaron: plan terapéutico empírico inicial, duración de la estadía hospitalaria, necesidad de procedimientos quirúrgicos, complicaciones (sepsis, shock séptico, trombosis venosa profunda, osteomielitis crónica) y letalidad.

Las fuentes de datos fueron la historia clínica y los registros del Laboratorio de Bacteriología del HP-CHPR.

Para la descripción de variables discretas se utilizó frecuencias absolutas y relativas, y para las variables continuas mediana y rango.

Se comparó la frecuencia de los microorganismos aislados en el período previo y mismo centro asistencial con los hallados en este período ${ }^{4}$.

Para la comparación de grupos se utilizó test no paramétrico U de Mann-Whitney, de variables discretas test de chi cuadrado de Pearson y de Fisher. Se consideró significativo $\mathrm{p}<0,05$. Para el procesamiento se utilizó el programa Epi- Info 7.0 (2012).

El estudio fue autorizado por la Dirección y el Comité de Ética del HP-CHPR. Se garantizó la confidencialidad y privacidad de los datos.

\section{Resultados}

En el período 2009-2015 se estudiaron 335 pacientes con sospecha de IOA. Se aisló algún microorganismo en 113 (34\%). Se incluyeron en el estudio 86 niños (76\%) con OIA confirmada; uno de ellos presentó dos IOA separados por cuatro meses en diferentes localizaciones y fueron considerados episodios independientes. Se excluyeron 27 por no contar con el registro completo de la historia clínica.

La distribución anual de los casos incluidos fue 21 en el año 2009, 13 en 2010, 12 en 2011, 16 en 2012, 9 en 2013, 8 en 2014 y 8 en 2015 . 
La mediana de edad fue 7 años (rango 16 días a 14 años); 62 (72\%) de sexo masculino.

El tipo de IOA fue osteomielitis en 33 (38\%) casos (3 no hematógenas secundarias a fracturas expuestas), artritis en 30 (35\%), osteoartritis en 22 (25\%) y bursitis en 2 (2\%).

En relación a la topografía, $68(78 \%)$ correspondieron a miembros inferiores, $17(20 \%)$ a miembros superiores y $2(2 \%)$ a focos múltiples.

En todos los casos se realizó hemocultivos. Se aisló el mismo microorganismo en hemocultivo que en el tejido óseo y/o líquido articular en 20 (23\%). En el resto de los casos el hemocultivo fue estéril.

Se realizó cintigrama (centelleograma) óseo en 17 $(20 \%)$, resonancia magnética en $10(11 \%)$ y ambos estudios en 9 (10\%). Los hallazgos fueron compatibles con el diagnóstico en 30/36 (83\%).

En 83 de los 87 casos (95\%) en el cultivo inicial se aisló un solo microorganismo (Tabla 1). En los restantes cuatro se aislaron dos o más microorganismos (Tabla 2). Todos los pacientes con cultivos polimicrobianos eran previamente sanos, tres sufrieron politraumatismos con fracturas expuestas y el otro se trataba de un recién nacido que durante la hospitalización por una enfermedad respiratoria desarrolló sintomatología compatible con IOA.

Kingella kingae se aisló de una muestra de punción ósea en un niño de 6 meses, previamente sano, con artritis tibio-tarsiana y reactantes de fase aguda (leucocitosis y PCR) normales.

En la evolución, en cinco niños se aisló un microorganismo distinto al identificado en el cultivo inicial en muestra/s óseas o articulares obtenidas durante procedimientos quirúrgicos posteriores al primario. Los mismos correspondieron a Klebsiella pneumoniae productora de $\beta$-lactamasas de espectro extendido (n: 2), Pseudomonas aeruginosa (n: 1), Burkholderia cepacia (n: 1) y Acinetobacter baumannii (n: 1).

En relación a la susceptibilidad antimicrobiana, en $S$. aureus, fueron susceptibles a meticilina 47/58 (81\%) y a eritromicina $52 / 58(87 \%)$. Todos los aislados fueron susceptibles a gentamicina, clindamicina y cotrimoxazol. Presentaron resistencia inducible a clindamicina $3(5 \%)$. En la Figura 1 se muestra la evolución anual de la susceptibilidad de $S$. aureus a meticilina en el período estudiado.

Los aislados de $H$. influenzae y $K$. kingae no fueron productores de $\beta$-lactamasas.

Las enterobacterias aisladas en los cultivos primarios incluyeron Escherichia coli, Salmonella sp, K. pneumoniae, K. oxytoca, Serratia marcescens y Pantoea sp.

Al comparar la etiología de las IOA en los períodos 2009-2015 vs 2003-2005, se observa un aumento no significativo en la frecuencia de aislamiento de enterobacterias y una reducción significativa en la frecuencia de aislamiento de SARM-AC (13 vs 27\%) y Streptococcus pneumoniae (1 vs 14\%) (Tabla 3).
Tabla 1. Infecciones osteo-articulares mono-microbianas. Hallazgos microbiológicos en el cultivo primario. Niños hospitalizados. HP-CHPR 2009-2015 (n: 83)

$\begin{array}{llc} & \text { SASM* } & 47 \\ \text { Cocáceas grampositivas } & \text { SARM** } & 11 \\ & \text { E. faecalis } & 2 \\ & \text { S. pyogenes } & 6 \\ & \text { S. agalactiae } & 1 \\ & \text { S. dysgalactiae ss. equisimilis } & 1 \\ & \text { S. pneumoniae } & 1 \\ & \text { Salmonella sp } & 1 \\ \text { Enterobacterias } & \text { S. marcescens } & 1 \\ & \text { Pantoea sp } & 1 \\ \text { Bacilos gramnegativos exigentes } & \text { H. influenzae tipo b } & 2 \text { *** } \\ & \text { H. influenzae no tipificable } & 1 \\ & \text { K. kingae } & 1 \\ \text { Pasteurella sp } & 1 \\ \text { Bacilos gramnegativos no fermentadores } & \text { P. aeruginosa } & 6 \\ & \text { Total } & 83\end{array}$

Tabla 2. Infecciones osteo-articulares polimicrobianas. Hallazgos microbiológicos en el cultivo primario. Niños hospitalizados. HP-CHPR 2009-2015 (n: 4)

\begin{tabular}{cl}
\hline Caso & Microorganismos \\
1 & K. pneumoniae + Staphylococcus coagulasa negativa + Candida sp. \\
2 & K. oxytoca + E. faecalis + S. marcescens \\
3 & E. faecalis + S. marcescens \\
4 & E. gallinarum + E. coli \\
\hline HP CHPR: Hospital Pediátrico Centro Hospitalario Pereira Rossell. \\
\hline
\end{tabular}

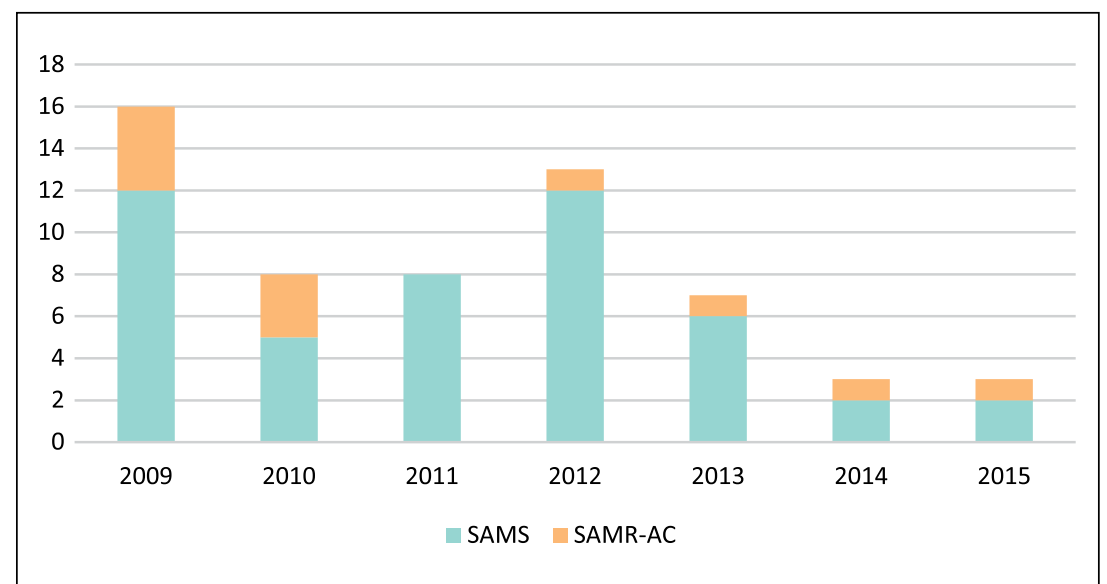

Figura 1. Infecciones osteo-articulares monomicrobianas causadas por Staphylococcus aureus. Evolución anual de la susceptibilidad de $S$. aureus a meticilina (n: 58). SASM: S. aureus sensible a meticilina. SARM-AC: $S$. aureus resistente a meticilina adquirido en la comunidad. 
Tabla 3. Infecciones osteo-articulares monomicrobianas. Comparación de hallazgos microbiológicos en el cultivo primario. Niños hospitalizados. HP-CHPR 2003-2005 vs 2009-2015

\begin{tabular}{lcccc}
\hline Etiología & $\begin{array}{c}\text { Período 2003-2005 } \\
\mathbf{n}(\%)\end{array}$ & $\begin{array}{c}\text { Período 2009-2015 } \\
\mathbf{n}(\%)\end{array}$ & $\mathbf{p}$ \\
\hline SASM $^{*}$ & $23(41)$ & $47(57)$ & NS \\
\hline SARM-AC** & $15(27)$ & $11(13)$ & $<0,05$ \\
\hline S. pneumoniae & $8(14)$ & 1 & $(1)$ & $<0,01$ \\
H. influenzae tipo b & 0 & 2 & $(2)$ & NS \\
K. kingae & 0 & 1 & $(1)$ & NS \\
Otros & $10(18)$ & $22(26)$ & NS \\
Total & $56(100)$ & $83(100)$ & \\
\hline
\end{tabular}

HP CHPR: Hospital Pediátrico Centro Hospitalario Pereira Rossell. SASM: S. aureus sensible a meticilina. SARM-AC: $S$. aureus resistente a meticilina adquirido en la comunidad.

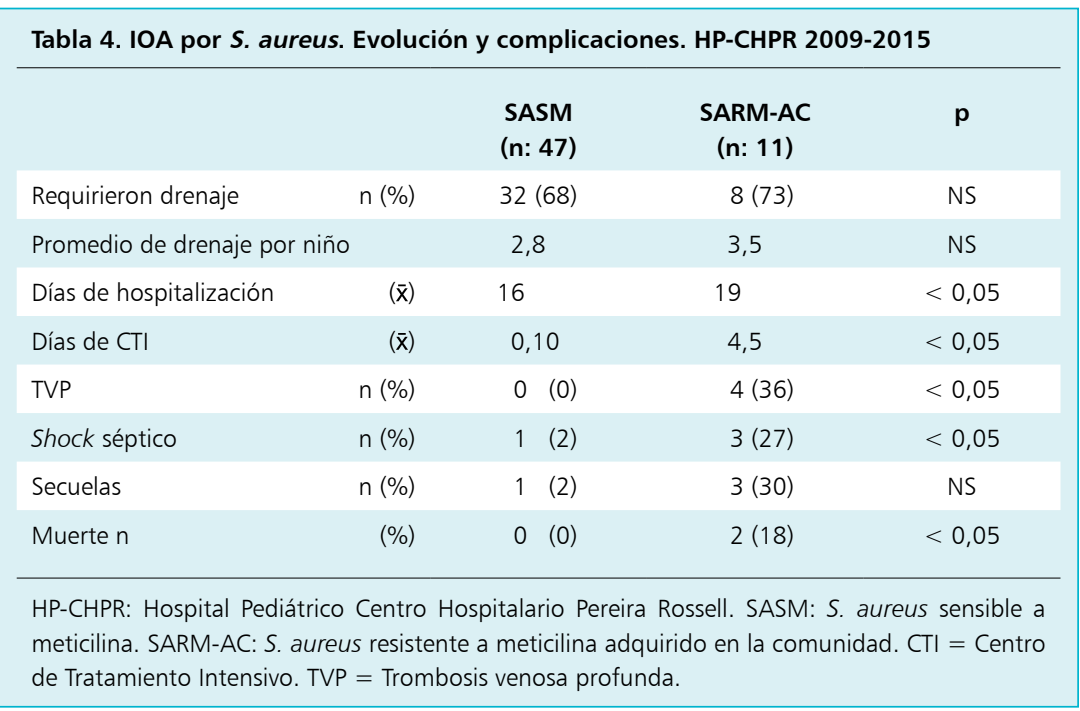

En $75(86 \%)$ se comenzó el tratamiento con clindamicina, asociada a otros antimicrobianos en 60: con gentamicina (n: 51), cefalosporinas (n: 5) o vancomicina (n: 4). En cuatro pacientes se utilizó cefalosporinas, en dos asociados a vancomicina. En tres recién nacidos se prescribió ampicilina asociada a cefalosporinas en dos casos y en uno a gentamicina.

Requirieron tratamiento quirúrgico 57 casos (66\%), promedio tres drenajes por niño.

La estadía hospitalaria promedio fue 17,3 días (rango 2-70). Requirieron ingreso a unidad de cuidado intensivo (UCI) 10 niños, promedio 11,8 días (rango 2-40).

El lactante en el que se aisló $K$. kingae permaneció internado durante 10 días, requirió tres drenajes quirúrgicos, presentando posteriormente evolución favorable, sin secuelas.

En la Tabla 4 se comparan las complicaciones, secuelas y letalidad de los niños con IOA por $S$. aureus, enterobacterias y bacilos gramnegativos no fermentadores. Cuatro presentaron manifestaciones clínicas de shock al ingreso; se aisló el agente causal en sangre en todos ellos.

Dos niños fallecieron en el período estudiado; en ambos se aisló SARM-AC en la punción ósea y en el hemocultivo. Se trataba de un varón de 11 años y una niña de 8 años, que habían presentado un traumatismo menor días previos y que al ingreso presentaron manifestaciones de shock séptico y trombosis venosa profunda. Se inició tratamiento empírico con clindamicina más vancomicina en ambos casos. Requirieron múltiples intervenciones quirúrgicas. En ambos la inestabilidad clínica impidió realizar estudios imagenológicos. No se realizaron autopsias clínicas.

\section{Discusión}

Coincidiendo con lo comunicado en el concierto internacional y con las series nacionales publicadas previamente, en esta serie las IOA afectan predominantemente a varones y se localizan preferentemente en los huesos largos de los miembros inferiores s,7,19-21. $^{4}$

$\mathrm{Su}$ diagnóstico y tratamiento oportuno puede mejorar el pronóstico a corto y largo plazo ${ }^{1}$. La confirmación etiológica implica el aislamiento del microorganismo causal en muestras de punción ósea y/o articular. En series extranjeras, la frecuencia de detección etiológica no supera el $50 \%{ }^{7}$; en esta serie fue de $38 \%$. La realización de dos hemocultivos se hace necesaria siendo el rendimiento de este examen en los niños con IOA superior a otras infecciones ${ }^{22}$. En el HP-CHPR se está introduciendo el uso de reacción de polimerasa en cadena (RPC) para aumentar la sensibilidad del diagnóstico etiológico. Este método no se encontraba disponible durante el período del presente estudio.

La utilización adecuada de los estudios microbiológicos permitiría conocer la etiología hasta en $80 \%$ de los $\operatorname{casos}^{23}$. Teniendo en cuenta la limitación de estos resultados, el diagnóstico de IOA también podría considerarse en aquellos casos en los cuales existe presunción clínica, reactantes de fase aguda elevados, estudio imagenológico compatible y/o hemocultivo positivo ${ }^{3,24}$.

El conocimiento de la etiología, formas de presentación clínica y evolución de las IOA resulta indispensable para mejorar el índice de sospecha y el abordaje diagnóstico y terapéutico.

Staphylococcus aureus continúa siendo el principal agente etiológico de las IOA en los niños hospitalizados 
en el HP-CHPR. La frecuencia global de aislamiento de este agente se ha mantenido estable desde el año 2003. Un resultado importante lo constituye la reducción significativa en la frecuencia de SARM-AC a lo largo del período. Este hallazgo había sido comunicado previamente por integrantes de este equipo de investigación, al analizar las IOA por $S$. aureus en niños hospitalizados en este centro entre los años 2009 y $2012^{4}$. Estudios con otros diseños serán necesarios para interpretar esta observación. Es posible que el uso universal de una pauta de tratamiento empírico para las infecciones causadas por SARM-AC explique en parte este hallazgo. Staphylococcus aureus sensible a meticilina (SASM) es actualmente la etiología más frecuente de las IOA en esta población. Sin embargo, SARM-AC representa $11,6 \%$ de los aislados por lo que se debe considerar siempre en el manejo antimicrobiano empírico inicial ante la sospecha diagnóstica ${ }^{9}$.

Resulta trascedente también destacar el registro, durante el período de estudio, de dos casos de IOA causadas por $H$. influenzae tipo b, a pesar de que esta etiología no represente un problema desde hace años en nuestro medio. La efectividad de la vacunación contra este agente no es $100 \%$, está asociada a la edad del niño, el número de dosis recibidas y las tasas de cobertura vaccinal en la comunidad y factores del hospedero como inmunodeficiencias primarias o secundarias. En esta serie, uno de los dos casos se podría tratar de una falla vaccinal, siendo ésta entendida como el aislamiento de $H$. influenzae tipo b en un líquido estéril transcurrida una semana o más de la segunda dosis de vacuna en un paciente menor de un año de edad o después de dos semanas de la última dosis de vacuna en pacientes de un año de edad o más ${ }^{25}$. Se necesitaría un análisis completo de la historia clínica en este caso para poder confirmarlo. En Uruguay, la cobertura global de la vacuna pentavalente, que incluye el componente $H$. influenzae tipo b, es 95\%. Sin embargo, según datos de la Comisión Honoraria para la Lucha Antituberculosa y Enfermedades Prevalentes, sólo 60\% de los niños reciben la segunda dosis antes de los 7 meses. Esto podría explicar la mayor exposición de niños susceptibles en un medio con circulación de este microorganismo. Es necesario mantener alerta ante este agente, sin olvidar que es causante de enfermedad invasora en niños pequeños, especialmente bajo 2 años de edad ${ }^{26}$.

Un cambio etiológico importante es la reducción significativa observada en el aislamiento de S. pneumoniae. Entre 2003 y 2005 S. pneumoniae era el segundo agente en frecuencia. Es altamente probable que esto se deba al impacto de la vacunación universal con vacuna conjugada anti-neumócoccica. Los beneficios sobre neumonía y meningitis han sido ampliamente documentados en nuestro país ${ }^{13,14}$. Estos resultados aportan al beneficio sobre otras enfermedades invasoras menos frecuentes, pero igualmente graves.
En esta era post-vaccinal, resulta importante mantener una vigilancia epidemiológica activa sobre la evolución de las enfermedades invasoras por $H$. influenzae tipo b y $S$. pneumoniae. La implementación de las vacunas conjugadas obliga al pediatra a conocer la situación epidemiológica del país y a realizar un análisis más profundo de cada caso, tratando de responder si se trata o no de un fallo vaccinal. En ese caso se impone el análisis de los determinantes del mismo, desde aspectos programáticos hasta factores individuales relacionados con la respuesta inmunológica.

En los últimos años, se ha comunicado un aumento en el número casos de IOA por $K$. kingae en distintas latitudes sustituyendo a $H$. influenzae como el principal bacilo gramnegativo responsable de artritis bajo 4 años de edad. En esta serie, en el período estudiado, se produjo el primer aislamiento de este agente en un lactante con osteo-artritis. El diagnóstico de esta infección representa un desafío. Los signos articulares pueden ser muy marcados pero las manifestaciones generales (fiebre, afectación del estado general) y la alteración de los reactantes de fase aguda (leucocitosis, PCR) generalmente son discretas. Existen dificultades para lograr el aislamiento de $K$. kingae debido a su crecimiento lento, su baja concentración en el foco de infección y su escasa capacidad de resistencia a condiciones adversas. Por lo tanto, con el fin de mejorar el rendimiento diagnóstico se puede realizar la inoculación de líquido sinovial o aspirado óseo en frascos de hemocultivo $^{27,28}$. La sospecha clínica de IOA por éste debe incluir en el plan terapéutico empírico inicial un antimicrobiano como una cefalosporina segunda generación ${ }^{17,29}$.

La mayoría de los casos de infección polimicrobiana en esta serie se produjo luego de fracturas abiertas contaminadas. La etiología polimicrobiana es lo habitual en las IOA cuyo mecanismo de producción es la contigüidad. Entre los patógenos involucrados se encuentran la microbiota cutánea normal que contamina la herida, microorganismos procedentes del suelo donde ocurrió el accidente o adquiridos tras efectuarse procedimientos quirúrgicos ${ }^{30}$.

El aumento en el número de aislados de bacilos gramnegativos resulta un hallazgo a vigilar. Su rol patógeno cobra mayor importancia en caso de infecciones intrahospitalarias y cuando existen co-morbilidades como diabetes mellitus o inmunodeficiencias. Pueden requerir además múltiples limpiezas quirúrgicas y estadías hospitalarias prolongadas, factores que predisponen a su vez al desarrollo de infecciones nosocomiales. En esta serie ningún niño presentó co-morbilidades. En su mayoría fueron aislados en pacientes politraumatizados formando parte de cultivos polimicrobianos o como agentes de infecciones intrahospitalarias.

En cinco niños se aisló durante su evolución clínica un microorganismo distinto al identificado en el cultivo 
inicial. Los mismos fueron bacilos gramnegativos no fermentadores y $K$. pneumoniae productoras de $\beta$-lactamasas de espectro extendido. Resulta necesario fortalecer la política de control y manejo de estas infecciones con el objetivo de disminuir el impacto de estos agentes que plantean dificultades en el tratamiento por los patrones de resistencia antimicrobiana.

Los patrones de susceptibilidad in vitro de los microorganismos más frecuentemente implicados en las IOA en esta población apoyan la vigencia de las pautas de tratamiento habitualmente recomendadas para el HPCHPR. Considerando la gravedad de las infecciones por SARM-AC, la terapia empírica inicial siempre debe cubrir la posibilidad de este agente, a pesar que su prevalencia viene en descenso ${ }^{31}$. La susceptibilidad de SARM-AC a macrólidos y clindamicina es elevada. No se detectó resistencia constitutiva a clindamicina y la resistencia inducible sólo se observó en tres cepas. Si bien la importancia clínica de la resistencia inducible a clindamicina no ha sido completamente establecida, constituye un fenómeno preocupante que debe vigilarse, ya que existen algunas comunicaciones de falla terapéutica ${ }^{32,33}$. Los hallazgos en esta serie apoyan el uso empírico de clindamicina en las IOA en niños a partir de dos meses de edad. El hallazgo de bacilos gramnegativos exigentes como $H$. influenzae y K. kingae en niños bajo 4 años de edad, obliga a considerar la asociación en estos grupos de cefalosporinas de segunda generación ${ }^{3,17}$. En lactantes bajo 2 meses se recomienda la asociación de cefalosporina de tercera generación más vancomicina, con o sin el agregado de un aminoglucósido ${ }^{3}$.

La proporción de niños que requirieron drenaje quirúrgico y el promedio de drenajes por niño fue elevada y similar en los niños con IOA por SARM-AC y SASM. Esto probablemente está vinculado con la conducta traumatológica frente a las complicaciones supurativas. Sin embargo, la estadía hospitalaria y en UCI fue más prolongada en los niños con SARM-AC. La frecuencia de secuelas también fue mayor, aunque no alcanzó significación estadística. La trombosis venosa profunda y shock séptico se asociaron a infección por SARM-AC.

Esta mayor gravedad de las infecciones por SARMAC también se reporta en otras series regionales ${ }^{34-36}$. Estudios microbiológicos señalan que la gravedad de $S$. aureus no es una característica exclusiva de las cepas resistentes a meticilina, si bien éstas parecen poseer más atributos de virulencia, como la producción de una proteína de unión al colágeno (CNA) y de una exotoxina la leucocidina de PantonValantine que destruye los neutrófilos del hospedero generando inmunocompromiso y aumentando el riesgo de coagulopatía intravascular. Conduce además a la producción de material purulento más aglutinado, difícil de drenar y erradicar, por lo que el abordaje quirúrgico generalmente es más complejo y frecuente $\mathrm{e}^{36-39}$. Las dos muertes se registraron también en este grupo etiológico.

\section{Conclusiones}

Staphylococcus aureus continúa siendo la etiología más frecuente de IOA. A pesar del descenso en la prevalencia de SARM-AC, las IOA causadas por este microorganismo siguen siendo graves, mortales y con mayores secuelas, por lo que las pautas nacionales de tratamiento en las IOA aún tienen vigencia en nuestro medio.

Para la pesquisa etiológica se requiere continuar con la práctica sistemática de obtener muestras óseas y/o de líquido articular.

Dado el aumento de los microorganismos gramnegativos, hay que tenerlos en cuenta al momento de indicar el tratamiento antimicrobiano, el especial en aquellos pacientes posibles de drenajes múltiples, fracturas expuestas u otras co-morbilidad.

Es destacable el impacto que ha tenido vacunación universal en el descenso de las infecciones invasoras por $S$. pneumoniae y $H$. influenzae tipo b.

Estos resultados apoyan la importancia de la vigilancia epidemiológica.

\section{Resumen}

Introducción: El agente etiológico más frecuente de las infecciones osteoarticulares (IOA) es Staphylococcus aureus. Luego de la introducción de nuevas vacunas la prevalencia de otros microorganismos ha cambiado. Objetivo: Describir la etiología y evolución de las IOA en niños internados en el Hospital Pediátrico del Centro Hospitalario Pereira Rossell entre los años 2009 y 2015. Pacientes y Métodos: Se identificaron los cultivos de muestras de líquido articular y punción ósea de niños con sospecha de IOA. Se analizaron sus características epidemiológicas, microorganismos aislados, exámenes complementarios, tratamiento inicial, complicaciones y días de hospitalización. Resultados: Se estudiaron 335 pacientes, se aisló microorganismo en 113. Se analizó 87 casos. Edad media 6,1 años. Correspondieron a $S$. aureus sensible a meticilina (n: 47), S. aureus resistente a meticilina adquirido en la comunidad (SARM-AC) (n: 11), Streptococcus pyogenes (n: 6), Streptococcus pneumoniae (n: 1), Haemophilus influenzae tipo b (n: 2), bacilos gramnegativos (n: 9): Kingella kingae (n: 1), otros (n: 6). En 4 casos se aisló más de un microorganismo. Las infecciones por SARM-AC fueron más graves. Discusión: Se observa un descenso de aislados de SARM-AC. Se destaca el impacto de la vacunación universal contra $S$. pneumoniae y $H$. influenzae tipo b. Se debe vigilar el aumento de aislamiento de bacilos gramnegativos. 


\section{Referencias bibliográficas}

1.- Kaplan S. Osteomielitis. En: Kliegman RM, Beherman RE, Jenson HB, Stanton BF, eds. Nelson Tratado de Pediatría. 19 ed. Barcelona: Elsevier 2013: 2471-5.

2.- Pääkkönen M, Peltola H. Bone and joint infections. Pediatr Clin North Am 2013; 60 (2): 425-36.

3.- Departamento de Pediatría. Facultad de Medicina. Atención Pediátrica. Normas Nacionales de Diagnóstico, Tratamiento, Prevención. Infección osteoarticular. 8va ed. Montevideo: Oficina del libro-FEFMUR 2014; 549: 59.

4.- Romero M C, Mas M, Giachetto G, Algorta G, Pírez M C, Cuneo A, et al. Etiología y presentación clínica de las Infecciones osteoarticulares en niños hospitalizados en Hospital Pediátrico del Centro Hospitalario Pereira Rossell 2003-2005. Rev Med Urug 2008; 24 (4): 238-45.

5.- Pandolfo S, Vomero A, Ambrosoni M, Zunino C, Algorta G, Giachetto G. Características de las infecciones osteoarticulares por Staphylococcus aureus en niños hospitalizados. Hospital Pediátrico del Centro Hospitalario Pereira Rossell 2009-2012. Arch Pediatr Urug 2013; 84 (S1): S42-S47.

6.- Gutiérrez K. Osteomyelitis. En: Long S, Pickering L, Prober C. Principles and Practice Infectious Diseases. 3 ed. Philadelphia: Elsevier, 2008: 474-82.

7.- Krogstad P. Osteomyelitis. En: Feigin RD, Cherry JD, Demmler-Harrison GL, Kaplan SL. Feigin and Cherry's: TextBook of Pediatric Infectious Diseases, 6 ed. Philadelphia: Elsevier, 2009: 725-42.

8.- Galiana A. infección por Staphylococcus aureus meticilino resistente adquirido en la comunidad. Arch Pediatr Urug 2003; 74 (1): 26-9.

9.- Giachetto G, Martínez A, Pírez M C, Algorta G, Banchero P, Camacho G, et al. Vigilancia en el uso de antibióticos en el Hospital Pediátrico del Centro Hospitalario Pereira Rossell: susceptibilidad antimicrobiana; gasto y consumo de antibióticos. Rev Med Urug 2003; 19 (3): 2008-15.

10.- Peltola H. Worldwide Haemophilus influenza type $b$ disease at the beginning of the $21 \mathrm{st}$ century: Global analysis of the disease burden 25 years after the use of the polysaccharide vaccine and a decade after the advent of conjugates. Clin. Microbiol. Rev 2000; 13 (2): 3012-7.

11.- Ruocco G, Curto S, Savio M, Laurani H, Frocht R. Vacunación contra Haemophilus influenzae tipo b en el Uruguay: experiencia e impacto. Rev Panam Salud Publica 1999; 5 (3): 197-9.
12.- Montano A, Algorta G, Pírez M C, Pascale A, Farcilli R, Ferrari A. Enfermedades invasivas por Haemophilus influenzae tipo b. Impacto de la vacunación en los niños que ingresan al Centro Hospitalario Pereira Rossell. Rev Med Urug 2001; 17: 166-70.

13.- Picón T, Alonso L, García Gabarrot G, Speranza N, Casas M, Arrieta F, et al. Effectiveness of the 7-valent pneumococcal conjugate vaccine against vaccine-type invasive disease among children in Uruguay: An evaluation using existing data. Vaccine 2013; 31 S3:C109-13. doi: 10.1016/j. vaccine.2013.01.059.

14.- Pírez M C, Algorta G, Cedrés A, Sobrero $H$, Varela A, Giachetto $G$, et al. Impact of universal pneumococcal vaccination on hospitalizations for pneumonia and meningitis in children in Montevideo, Uruguay. Pediatr Infect Dis J 2011; 30 (8). doi: 10.1097/ INF.0b013e3182152bf1.

15.- Hortal M, Sehabiague G, Camou T, Iraola I, Estevan M, Pujadas M. Pneumococcal pneumonia in hospitalized children and potential prevention with different vaccine formulations. J Pediatr. 2008; 152 (6): 850-3. doi: 10.1016/j.jpeds.2007.11.008.

16.- Machado K, López A, Pacheco H, Algorta G, Pírez M C. Características del empiema paraneumónico luego del inicio de la vacunación antineumocóccica. Centro Hospitalario Pereira Rossell, año 2010. Arch Pediatr Urug 2014; 85 (4): 212-9.

17.- Uría M, Pelufo G, Galazka G, Picón T, Giachetto G. Infección osteoarticular por Kingella kingae en niños. Descripción de casos clínicos. Arch Pediatr Urug 2012; 83 (4): 262-5.

18.- CLSI. Performance Standards for Antimicrobial Susceptibility Testing; Twenty-second Informational Supplement, CLSI document M100-S22. Wayne, PA: Clinical and Laboratory Standards Institute; 2012.

19.- Goergens E D, McEvoy A, Watson M, Barret I R. Acute osteomielytis and septic arthritis in children. J. Pediatr Child Health 2005; 41 (1,2): 59-62.

20.- Ferroni A. Epidemiology and bacteriological diagnosis of pediatrics acute osteoarticular infections. Arch Pediatr 2007; 14 Suppl 2 S91-6.

21.- Xue Ma X, Galiana A, Pedreira W, Mowszowicz M, Christophersen I, Machiavello S, et al. Community-acquired methicillin- resistant Staphylococcus aureus, Uruguay. Emerg Infect Dis 2005; 11 (6): 973-6.

22.- Kaplan S. Recent lessons for the management of bone and joint infections. J Infection 2014; 68, S51eS56.
23.- Isemberg H D. Clinical Microbiology Procedure Handbook. Washington: American Society of Microbiology, 1994.

24.- Peltola H, Pääkkönen M. Acute osteomyelitis in children. N Engl J Med 2014; 370: 352-60.

25.- Heath P T, Booy R, Griffiths H, Clutterbuck E, Azzopardi H J, Slack M P, et al. Clinical and immunological risk factors associated with Haemophilus influenzae type b conjugate vaccine failure in childhood. Clin Infect Dis 2000; 31: 973-809.

26.- WHO. Weekly epidemiological record. Haemophilus influenzae type b (Hib) vaccination. Position Paper 2013; 39 (88): 413-8.

27.- Lebel E, Rudensky B, Karasik M, Itzchaki M, Schlesinger Y. Kingella kingae infections in children. J Pediatr Orthopaedics 2006; 15: 289-95.

28.- Etxbarría I, Goikoetxea X, Sanado L. Infecciones osteoarticulares en niños por Kingella kingae. Rev Patol Aparato Locomotor 2006; 4 (3): 187-92.

29.- Budnik I, Porte L, Arce J, Vial S, Zamorano J. Espondilodiscitis causada por Kingella kingae en pediatría: reporte de un caso. Rev Chil Infect 2011; 28 (4): 369-73.

30.- Berbari E, Steckelberg J, Osmon D. Osteomielitis. En: Mandell Douglas Bennett's. Enfermedades infecciosas. Principios y Práctica. $7^{\mathrm{a}}$ ed Barcelona: Elsevier, 2012: 1466-70.

31.- Pääkkönen M. Management of osteoarticular infections caused by Staphylococcus aureus is similar to that of other etiologies analysis of 199 staphylococcal bone and joint infections Pediatr Infect Dis J 2012; 31: 436-8.

32.- Martínez-Aguilar G, Hammerman W A Mason E, Kaplan S L. Clindamycin treatment of invasive infections caused by community -acquired, methicillin- resistant and methicillin susceptible Staphylococcus aureus in children. Pediatr Infect Dis J 2003; 2 (7): 852-4.

33.- Szczesiul J, Shermock K, Murtaza U, Siberry G K. No decrease in clindamycin susceptibility despite increased use of clindamycin for pediatric communityassociated methicillin resistant Staphylococcus aureus skin infections. Pediatr Infect Dis J 2007; 26 (9): 852-4.

34.- Rosanova M, Berberian G, Bologna R, Giménez S, Sarkis C, Buchovsky A, et al. Estudio descriptivo de infecciones osteo-articulares en niños en tiempos de Staphylococcus aureus resistente a meticilina de la comunidad (SARM-AC). Rev Chilena Infectol 2015; 32 (3): 321-5.

35.- Acuña M, Benadof D, Jadue C, Hormazábal J, Alarcón P, Contreras J, et al. Staphylococcus 
aureus resistente a meticilina asociado a la comunidad (SARM-AC): comunicación de los primeros cuatro casos pediátricos descritos en Hospital de Niños Roberto del Río. Rev Chilena Infectol 2015; 32 (3): 350-6.

36.- Guillén R, Carpinelli L, Rodríguez F, Castro H, Quiñónez B, Campuzano A, et al. Staphylococcus aureus adquiridos en la comunidad: caracterización clínica, fenotípica y genotípica de aislados en niños paraguayos. Rev Chilena Infectol 2016; 33 (6): 609-18.

37.- Telechea H, Speranza N, Lucas I, Santurio A, Giachetto G, Algorta G, et al. Evolución en el consumo de antibiótico y de la susceptibilidad antimicrobiana en el Centro Hospitalario Pereira Rossell en la era de Staphylococcus aureus resistente a meticilina. Rev Chil Infectol 2009; 26 (5): 413-9.

38.- Mitchell P D, Hunt D M, Lyall H, Nolan
M, Tudor-Williams G. Panton Valentine leucocidin secreting Staphylococcus aureus causing severe musculoskeletal sepsis in children: a new threat. J Bone Joint Surg Br 2007; 89 (9): 1239-42.

39.- Pardo L, Vola M, Macedo Viñas M, Machado V, Cuello D, Mollerach M, et al. Community associated methicillin-resistant Staphylococcus aureus in children treated in Uruguay. J Infect Dev Ctries 2013; 7 (1): 10-6. 